\title{
Plasma MASP-1 concentration and its relationship to recovery from coronary artery lesion in children with Kawasaki disease
}

\author{
Rui-Xia Song ', Qing-Mei Zou ${ }^{2}$, Xiao-Hui Li', Nan-Ping Xu², Ting Zhang ${ }^{3}$, Jin Fu ${ }^{4}$ and Xiao-Dai Cui ${ }^{4}$
}

\begin{abstract}
BACKGROUND: This study investigated prognostic factors for early recovery of coronary artery lesion (CAL) in children with Kawasaki disease (KD).

METHODS: Patients hospitalized for KD were enrolled less than 2 wk from the onset of illness and divided into two groups: $\mathrm{KD}$ with CAL and KD without CAL. The CAL group was further divided into two subgroups according to the degree of $\mathrm{CAL}$ : mild $(n=31)$ and moderate/severe $(n=6)$ and further divided into two subgroups according to the age: younger than $1 \mathrm{y}$ $(n=9)$ and older than 1 y $(n=28)$. Lectin pathway-related factors MASP-1, CD59, and C5b-9 were measured, along with C-reactive protein, white blood cell counts, erythrocyte sedimentation rate, and platelet count. Patients were followed up for 3 mo. Correlation between the measured factors and the length of time of recovery from CAL was analyzed.
\end{abstract}

RESULTS: Plasma concentrations of MASP-1 in the CAL group were significantly lower than those without CAL. MASP-1 and gender positively correlated with the recovery time of CAL. There was no difference in MASP-1 between mild and moderate/severe CAL. At 3-mo follow-up, there was a positive correlation between plasma MASP-1 concentration and recovery time of the patients with CAL older than $1 \mathrm{y}$.

CONCLUSION: Plasma MASP-1 concentration at the early stage of $\mathrm{KD}$ is predictive of length of time of recovery from CAL.

K awasaki disease $(\mathrm{KD})$, also known as mucocutaneous lymph node syndrome, is an acute nonspecific systemic vascular inflammatory syndrome occurring mainly in children under $5 \mathrm{y}$ of age. A considerable volume of research has shown that the pathological process of $\mathrm{KD}$ is characterized by immune activation and immune vasculitis of the vascular endothelial cells (1). KD mostly affects the small and mediumsized arteries, especially the coronary artery (2), resulting in coronary artery lesion (CAL), such as dilation, aneurysm formation, or advanced stenosis, and it is the leading cause of death in children with KD (3). It has been reported that 15$25 \%$ of untreated KD patients may develop CAL (4). Although treatment with intravenous immunoglobulin within $10 \mathrm{~d}$ of onset of fever reduces the incidence of CAL, 5-8\% of patients still develop it. The degree of CAL varies considerably between individuals, ranging from mild to moderate ectasia of the coronary artery to giant aneurysm formation. The recovery time of CAL also differs between patients. There is a very litthe information about the length of time for which different degrees of CAL persists. Little is known on the relationship between the complement system and the recovery time of $\mathrm{CAL}$ resulting from $\mathrm{KD}$. Our previous study indicated that the lectin pathway of the complement system is activated in $\mathrm{KD}$. CAL is associated with endothelial cell dysfunction induced by T-cell activation (5). Endothelial cells are a source of complement-related factors and complement regulatory factors (6), and MASP-1 activates endothelial cells directly, which then activates the complement lectin pathway (7). Endothelial cell dysfunction (8) and complement-dependent cytotoxicity exist in the course of KD (9). Cheung et al. (10) reported that mannose-binding lectin genotype modulates arterial stiffness in children after KD. Based on these evidences, we speculated that plasma MASP-1 concentration may be associated with KD-related CAL. This study was designed to explore this relationship, in order to inform active intervention and future targeted therapies to prevent the development of CAL sequelae in children with KD.

\section{RESULTS}

\section{Two-Dimensional Echocardiography}

From 85 patients, 37 were assigned to the CAL group and 48 to the NCAL group. Of the CAL group, 31 patients presented with mild coronary artery dilatation and 6 patients with moderate/severe coronary artery dilatation. Of the CAL patients, 14 had left coronary artery dilatation (38\%), 9 had right coronary artery dilatation $(24 \%)$, and 14 had double coronary dilatation (38\%).

\section{Plasma Concentrations of MASP-1, C5b-9, and CD59 for Each Group}

The plasma MASP-1 concentration was significantly lower in the CAL group than that of healthy control and febrile control

\footnotetext{
The first two authors contributed equally to this work and should be considered as co-first authors.

'Department of Cardiovascular Diseases, Children's Hospital Affiliated to the Capital Institute of Pediatrics, Beijing, China; ${ }^{2}$ Department of Emergency, Children's Hospital of Jiangxi Province, Nanchang, Jiangxi, China; ${ }^{3}$ Central Laboratory of Infection and Immunity, Capital Institute of Pediatrics, Beijing, China; ${ }^{4}$ Clinical Center Laboratory, Capital Institute of Pediatrics, Beijing, China. Correspondence: Xiao-Hui Li (Ixhmaggie@126.com)

Received 8 March 2015; accepted 31 July 2015; advance online publication 2 December 2015. doi:10.1038/pr.2015.223
} 
groups $(4.84 \pm 2.11 \mathrm{vs} .6 .55 \pm 2.73 \mu \mathrm{g} / \mathrm{ml}$ and $5.73 \pm 2.36 \mu \mathrm{g} / \mathrm{ml}$; all $P<0.05)$. The plasma MASP-1 concentration was significantly lower in the CAL group than that of NCAL group $(4.84 \pm 2.11$ vs. $5.88 \pm 2.38 \mu \mathrm{g} / \mathrm{ml} ; P<0.05)$ (Figure 1a). The plasma C5b-9 concentration was significantly lower in the CAL group than that of healthy control group $(0.57 \pm 0.59$ vs. $1.38 \pm 1.37 \mu \mathrm{g} / \mathrm{ml} ; P<0.05)$; however, there was no significant difference between CAL group and febrile control group $(0.57 \pm 0.59$ vs. $0.81 \pm 0.83 \mu \mathrm{g} / \mathrm{ml} ; P>0.05)$. There was no significant difference in plasma concentration of C5b-9 between CAL and NCAL groups $(0.57 \pm 0.59$ vs. $0.61 \pm 0.66$ $\mu \mathrm{g} / \mathrm{ml} ; P>0.05$ ) (Figure $\mathbf{1 b}$ ). The plasma CD59 concentration was significantly lower in the CAL group than that of NCAL group $(0.2 \pm 0.15$ vs. $0.4 \pm 0.35 \mu \mathrm{g} / \mathrm{ml} ; P<0.05)$; however, there was no significant difference among CAL, healthy control, and febrile control groups $(0.2 \pm 0.15$ vs. $0.21 \pm 0.09$ $\mu \mathrm{g} / \mathrm{ml}$ and $0.2 \pm 0.15$ vs. $0.22 \pm 0.1 \mu \mathrm{g} / \mathrm{ml}$; all $P>0.05)$ (Figure 1c).
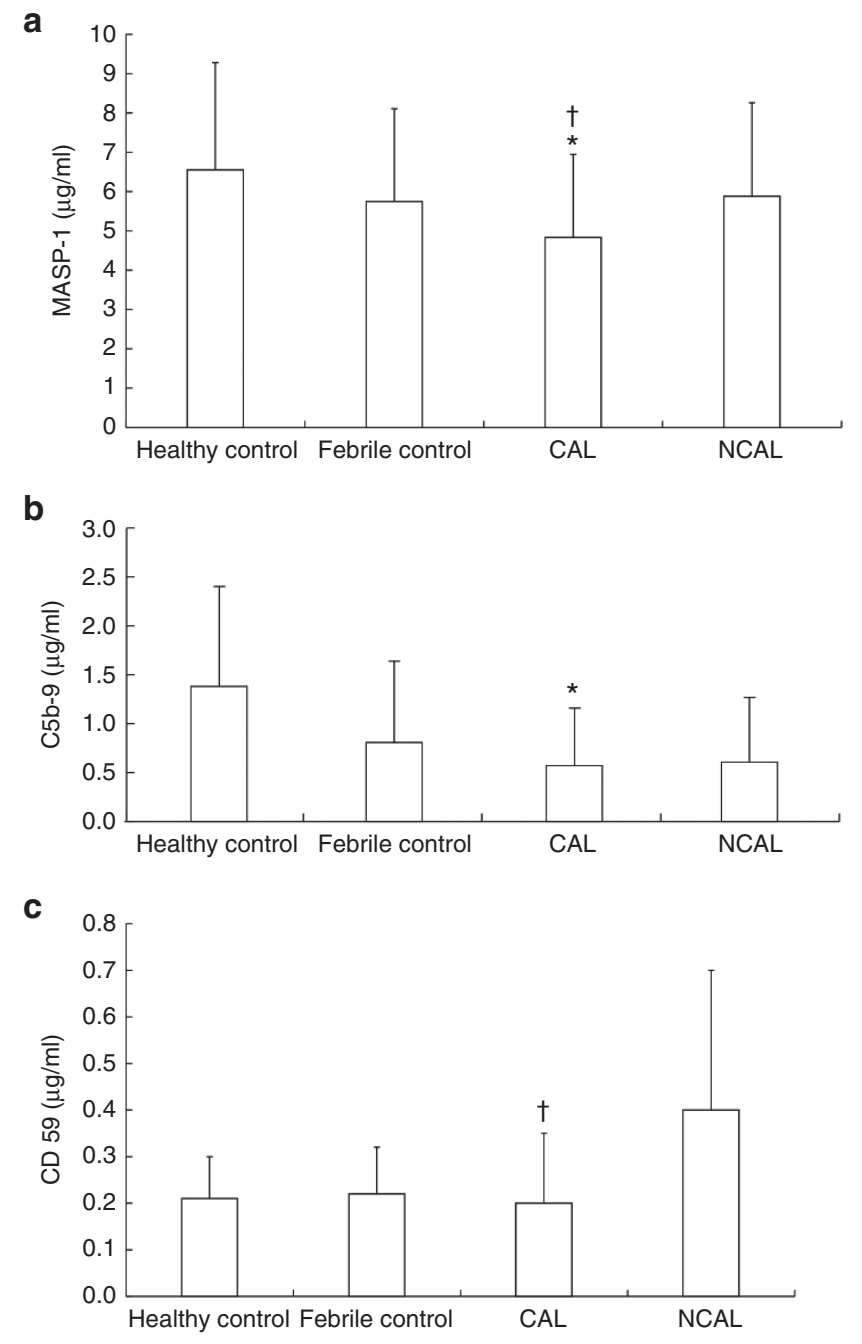

Figure 1. Plasma concentration of MASP-1, C5b-9, and CD59 in each group. (a) Plasma concentration of MASP-1, (b) plasma concentration of C5b-9, and (c) plasma concentration of CD59. $P<0.05$ was considered as statistically significant. ${ }^{\dagger} P<0.05$, CAL group vs. NCAL group; ${ }^{*} P<0.05$, CAL group vs. healthy control group and/or febrile control group. CAL, coronary artery lesion; NCAL, non-coronary artery lesion.
Plasma Concentrations of MASP-1, C5b-9, and CD59 for Each Subgroup

There was no significant difference in plasma concentration of MASP-1 between the mild and moderate/severe CAL subgroups $(4.70 \pm 2.13$ vs. $5.53 \pm 2.07 \mu \mathrm{g} / \mathrm{ml} ; P>0.05)$ (Figure $2 \mathrm{a}$ ). There was no significant difference in plasma concentrations of C5b-9 and CD59 between the mild and moderate/severe CAL subgroups $(0.59 \pm 0.98$ vs. $1.3 \pm 1.3 \mu \mathrm{g} / \mathrm{ml} ; P>0.05$ and $0.20 \pm 0.16$ vs. $0.23 \pm 0.15 \mu \mathrm{g} / \mathrm{ml} ; P>0.05$ ) (Figure $2 \mathrm{~b}, \mathrm{c}$ ).

\section{Changes in Inflammatory Markers White Blood Cell, C-Reaction} Protein, Erythrocyte Sedimentation Rate, and Platelet Count in the Early Stage of KD

Platelet count (PLT) was significantly higher in the CAL group compared with that of the NCAL group $\left(405.96 \pm 118.79 \times 10^{9} / 1\right.$ vs. $\left.345.66 \pm 87.83 \times 10^{9 / 1} ; \quad P<0.05\right)$ in early stage of $\mathrm{KD}$ (Figure 3a). However, there was no significant difference in white blood cell (WBC), C-reaction protein (CRP), and

a

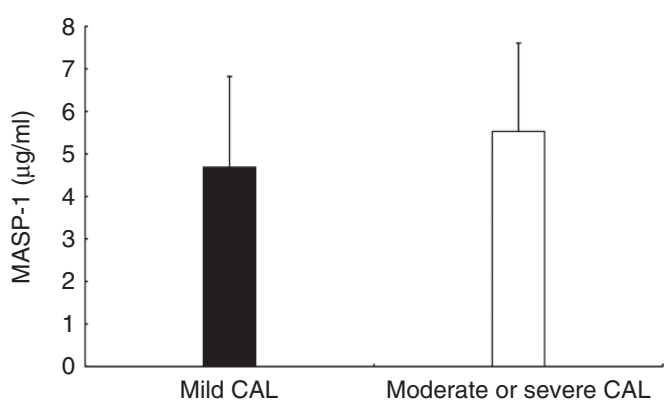

b

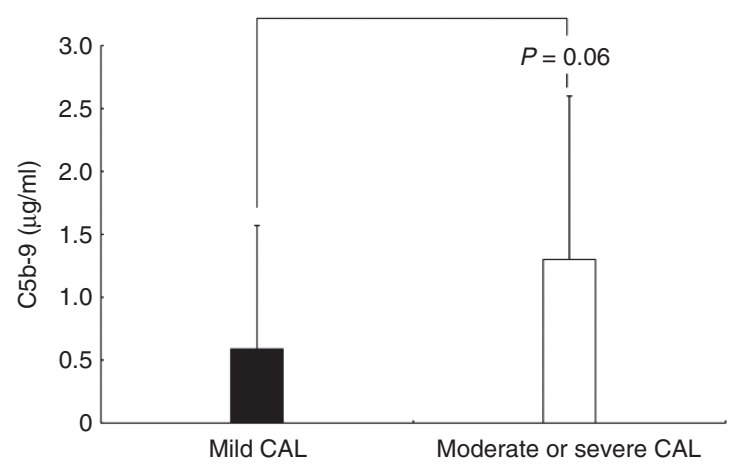

C

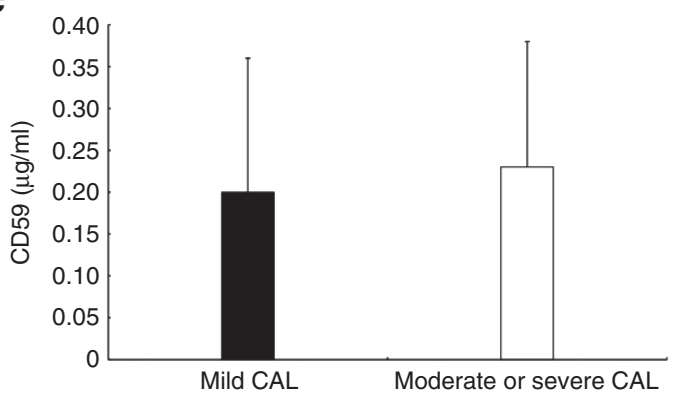

Figure 2. Plasma concentration of MASP-1, CD59, and C5b-9 in each subgroup. (a) Plasma concentration of MASP-1, $P>0.05$, (b) plasma concentration of C5b-9, $P=0.06$, and (c) plasma concentration of CD59, $P>0.05$. $P<0.05$ was considered as statistically significant. CAL, coronary artery lesion. 
a

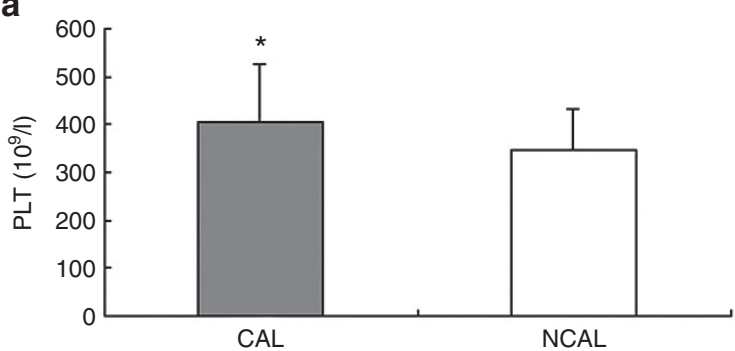

C

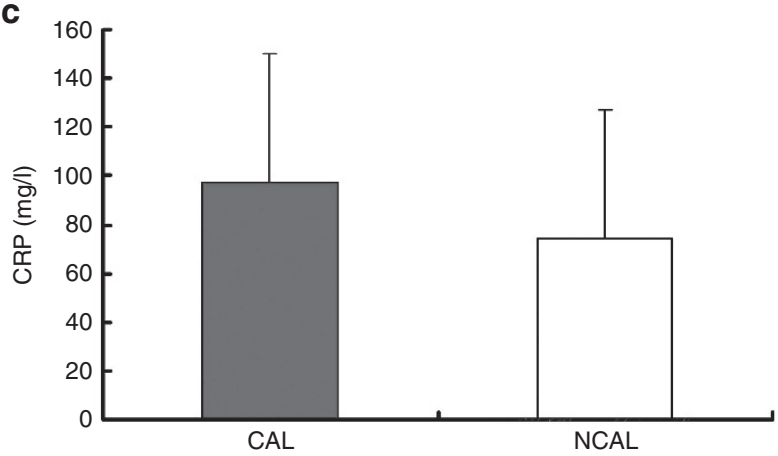

b

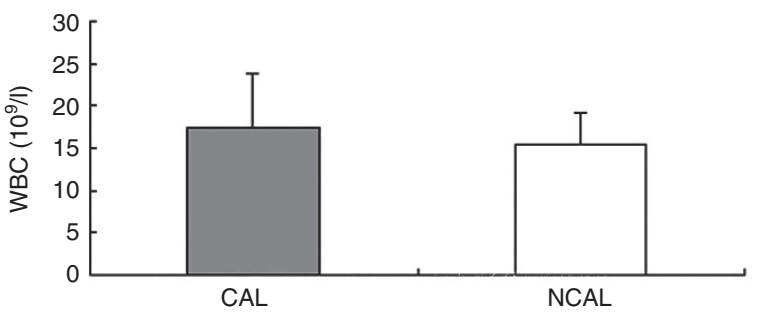

d

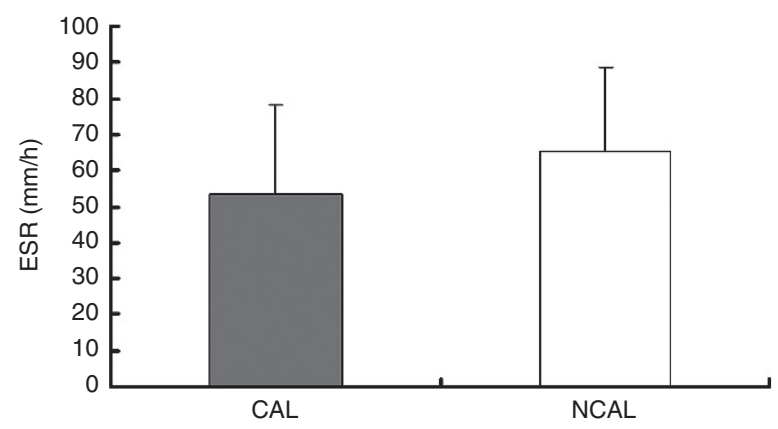

Figure 3. Changes of inflammatory factors WBC, CRP, ESR, and PLT in early stage of KD. (a) Plasma levels of PLT, (b) plasma levels of WBC, (c) plasma levels of CRP, and (d) plasma levels of ESR. $P<0.05$ was considered as statistically significant. CAL, coronary artery lesion; CRP, C-reaction protein; ESR, erythrocyte sedimentation rate; KD, Kawasaki disease; NCAL, non-coronary artery lesion; PLT, platelet count; WBC, white blood cell.

erythrocyte sedimentation rate (ESR) between the CAL and NCAL groups (WBC: $17.49 \pm 6.44 \times 10^{9} / \mathrm{lvs} .15 .44 \pm 3.78 \times 10^{9} / 1$, $P>0.05$; CRP: $(99.7,90.4)$ vs. $(60.45,71.5) \mathrm{mg} / \mathrm{l}, P>0.05$; ESR: $53.13 \pm 25.22$ vs. $65.16 \pm 23.69 \mathrm{~mm} / \mathrm{h}, P>0.05)$ (Figure $3 \mathrm{~b}-\mathrm{d}$ ).

\section{Follow-up Results}

Among patients with CAL, plasma MASP-1 concentration was significantly higher in patients whose coronary artery remained abnormal for longer than 3 mo compared with those whose coronary artery returned to normal within 3 mo (5.14 \pm 1.86 vs. $3.83 \pm 1.69 \mu \mathrm{g} / \mathrm{ml}, P<0.05$ ) (Figure 4a). ESR was also significantly higher in patients whose coronary artery remained abnormal for longer than $3 \mathrm{mo}(66.55 \pm 25.83$ vs. $45.5 \pm 12 \mathrm{~mm} / \mathrm{h}, P<0.05)$ (Figure 4b). However, there were no significant differences in WBC, CRP, and PLT between patients whose coronary artery returned to normal within 3 mo and those whose coronary artery remained abnormal for more than $3 \mathrm{mo}$ (WBC: $19.28 \pm 4.30 \times 10^{9} / 1$ vs. $17.58 \pm 2.35 \times 10^{9} / 1, \quad P>0.05$; CRP: $100.03 \pm 62.44$ vs. $102.13 \pm 32.52 \mathrm{mg} / \mathrm{l}, P>0.05$; PLT: $426.09 \pm 142.75 \times 10^{9} / 1$ vs. $390.25 \pm 103.88 \times 10^{9} / 1, P>0.05$ ) (Figure $4 c-e$ ).

\section{Relationship Between the Plasma Concentration of MASP-1,} Inflammatory Factors, Demographic Factors, and the Recovery Time of CAL

There was positive correlation between plasma MASP-1 concentration and the recovery time of the mild CAL subgroup $(r=0.46, P<0.05)$ (Figure 5a). There was positive correlation between plasma MASP-1 concentration and the recovery time of the older than age $1 \mathrm{y}$ subgroup $(r=0.76, P<0.05)$. There was no obvious correlation between WBC, CRP, ESR, PLT and the recovery time of CAL (WBC: $r=-0.28, P=0.24$;
CRP: $r=-0.06, P=0.78$; ESR: $r=0.44, P=0.06$; PLT: $r=-0.014$, $P=0.95$ ) (Figure $5 \mathrm{~b}-\mathrm{e}$ ). Multivariate regression showed that MASP-1 and gender are significantly positively correlated with the recovery time of CAL $(P<0.05)$ (Table 1$)$.

\section{DISCUSSION}

$\mathrm{KD}$ is a type of vasculitis, induced by an immune inflammatory reaction of unknown etiology, the incidence of which has gradually increased in recent years $(11,12)$. The main complication from KD is cardiovascular injury, particularly CAL, which can present as coronary artery dilation, aneurysm, or stenosis. The complement system plays an important anti-inflammatory role in the cardiovascular system (13). An excessively activated complement system leads to a cascade of enzymatic reactions resulting in injury to the host's own tissue. Studies reported that mannose-binding lectin is involved in the pathogenesis of the disease (14), and mannose-binding lectin genotype was associated with CALs in KD at various ages $(15,16)$. MASP-1, a key protease in the lectin pathway of the complement system, can activate vascular endothelial cells by protein kinase receptor-4, then activate neutrophils to play defensive role (17). MASP-1 is related to the structure and function of thrombin, which promotes leukocyte adhesion and aggregation by transformation of the vascular endothelial cell morphology and permeability, resulting in the formation of thrombus after coronary artery injury (18).

Our study showed that plasma MASP-1 concentration is significantly lower in patients with KD with CAL than in those without CAL, although plasma MASP-1 concentration is irrelevant to the severity of CAL. It also indicated that plasma MASP-1 concentration positively correlates with the recovery 


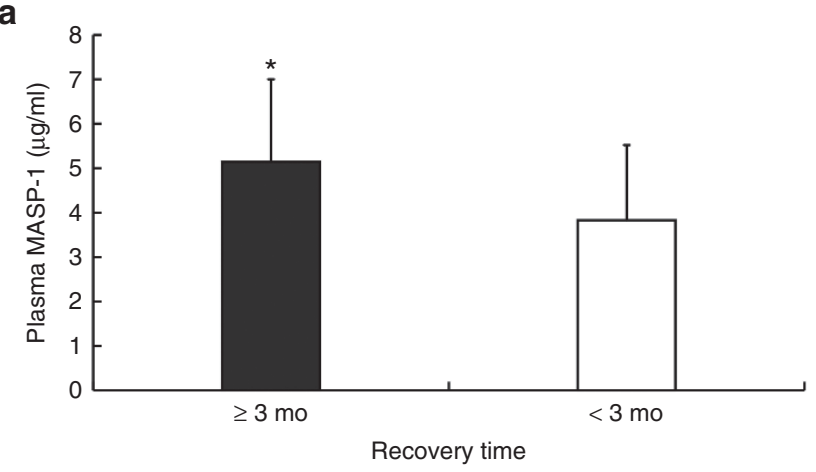

C

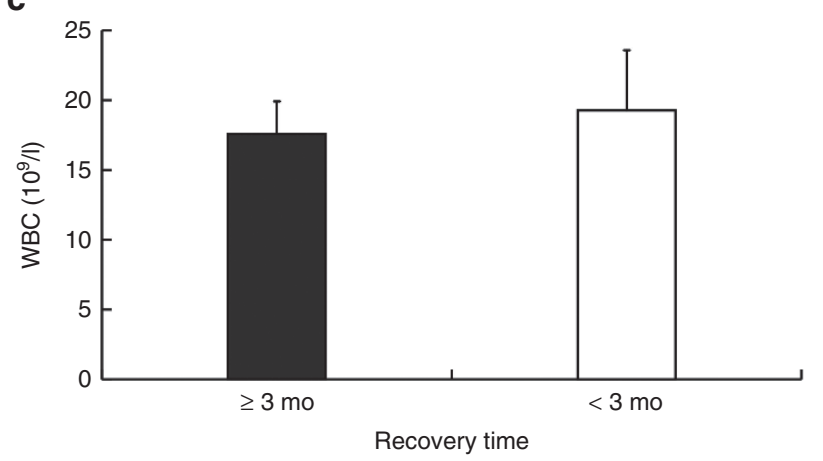

b

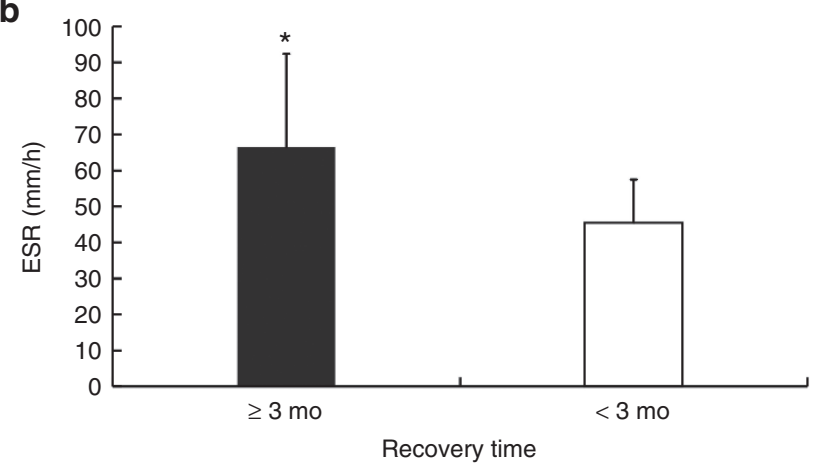

d

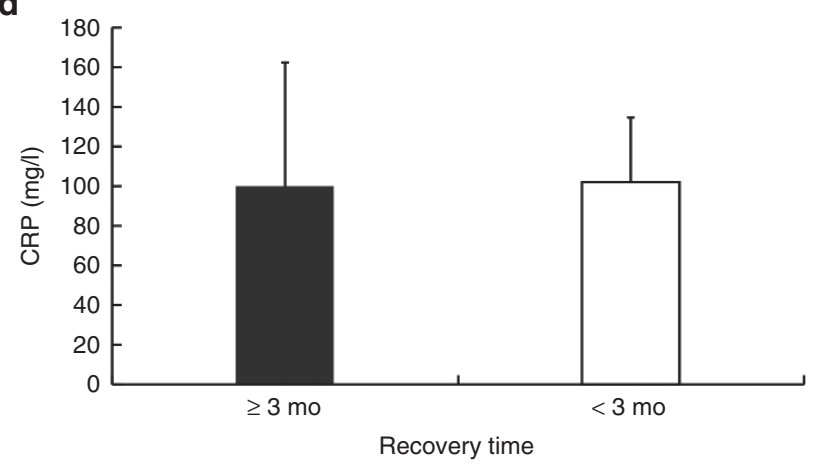

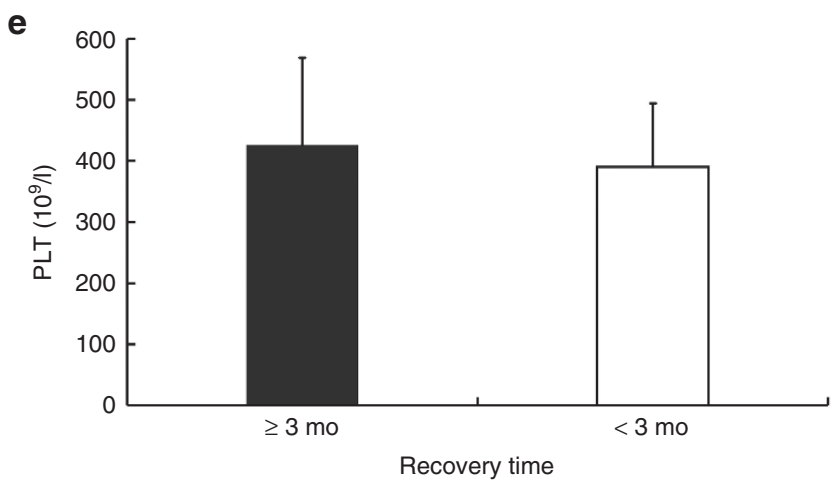

Figure 4. Recovery time and its relationship to MASP-1, ESR, WBC, CRP, and PLT. (a) Plasma MASP-1 concentration for recovery time from coronary artery lesion. (b) Plasma ESR concentration for coronary artery recovery time. (c) Plasma WBC concentration and coronary artery lesion recovery time. (d) Plasma CRP concentration and coronary artery lesion recovery time. (e) Plasma PLT concentration and coronary artery lesion recovery time. ${ }^{*} P<0.05$, coronary artery remained abnormal for longer than 3 mo vs. coronary artery returned to normal within $3 \mathrm{mo} . P<0.05$ was considered as statistically significant. CRP, C-reaction protein; ESR, erythrocyte sedimentation rate; PLT, platelet count; WBC, white blood cell.

time of coronary artery injury in $\mathrm{KD}$ patients older than $1 \mathrm{y}$, that is, when MASP-1 plasma concentration is increased, coronary artery restoration is delayed.

Turner (19) reported that plasma MASP-1 concentration lowers in the course of inflammatory disease, and Frauenknecht et al. (20) revealed that plasma MASP-1 concentration decreases in the acute stage of stroke; these findings are in accordance with our research. Studies by Jani et al. (21) indicate that MASP-1 directly activates human umbilical vein endothelial cells, exerting an anti-inflammatory effect by secreting IL-6 and IL-8. Thus, it is speculated that MASP-1 is activated in the early stage of $\mathrm{KD}$, provoking a vascular inflammatory reaction and resulting in the consumption of MASP-1. A considerable body of literature shows that KD patients with CAL experience more severe vasculitis than patients without
CAL (22), explained by a lower plasma MASP-1 concentration. This suggests that the presence of low plasma MASP-1 concentration can be considered a risk factor and experimental biomarker for CAL in KD.

Recent animal studies into the effects of the lectin pathway on ischemia-reperfusion injury, with the lectin pathway targeted by MASP-1 knockout and anti-MASP-1 monoclonal antibodies, indicate that MASP-1 exerts protective effects on myocardial and cerebral ischemia (23). Our study found that plasma MASP-1 concentration over a specific range prolonged the recovery time of CAL, explained by excessively activated MASP-1 exerting protective effects and simultaneously leading to immune injury. Endo et al. (24) demonstrated that MASP-1 is activated in the Henoch-Schonlein purpura, resulting in Henoch-Schonlein purpura nephritis due to glomerular 
a

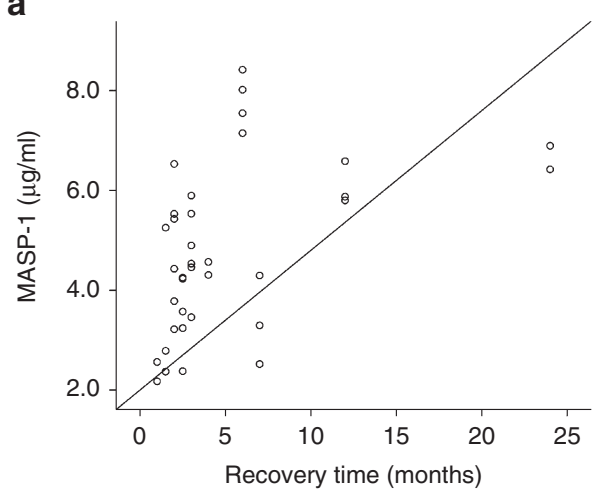

C

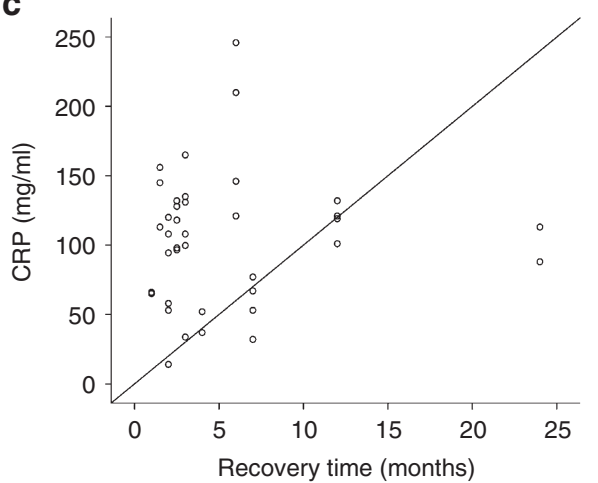

b

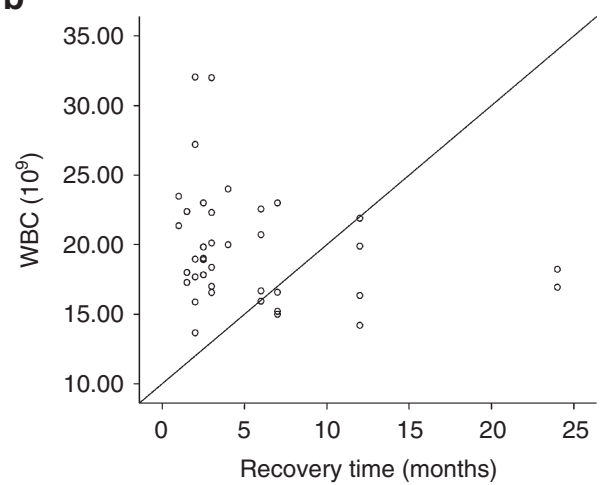

d

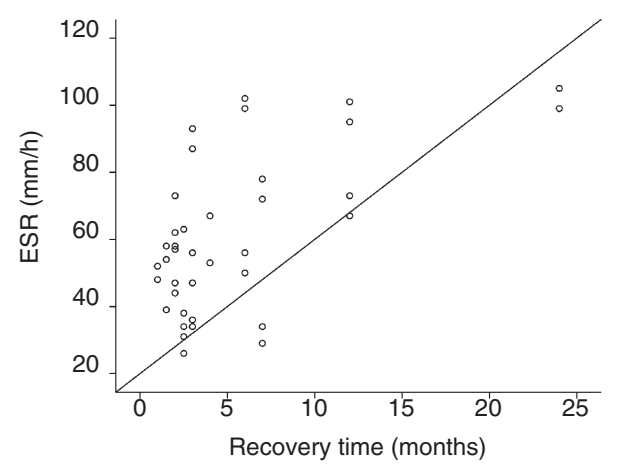

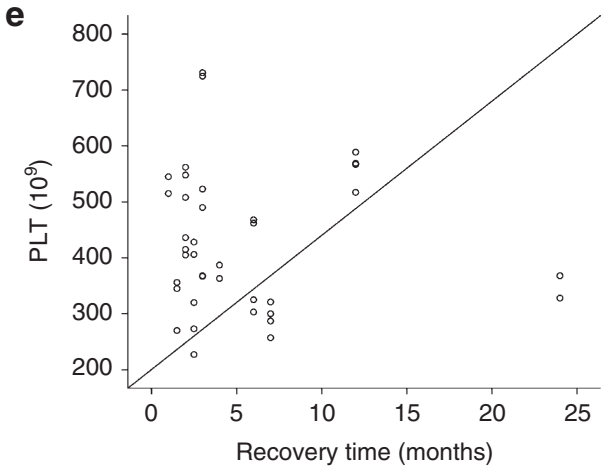

Figure 5. Correlation between the plasma concentration of MASP-1, inflammatory factors, demographic factors, and the recovery time of CAL. (a) Correlation between plasma MASP-1 concentration and the recovery time from CAL in KD patients. (b) Correlation between the count of WBC and the recovery time from CAL in KD patients. (c) Correlation between CRP and the recovery time from CAL in KD patients. (d) Correlation between ESR and the recovery time from CAL in KD patients. (e) Correlation between PLT and the recovery time from CAL in KD patients. CAL, coronary artery lesion; CRP, C-reaction protein; ESR, erythrocyte sedimentation rate; KD, Kawasaki disease; PLT, platelet count; WBC, white blood cell.

Table 1. Multivariate regression analysis of the plasma concentration of MASP-1, WBC, CRP, ESR, PLT, and the recovery time of CAL in KD

\begin{tabular}{lccccc}
\hline Variables & \multicolumn{1}{c}{$B$} & SE & \multicolumn{1}{c}{ OR } & \multicolumn{1}{c}{$95 \% \mathrm{Cl}$} & $P$ value \\
\hline MASP-1 & 0.726 & 0.739 & 2.066 & $0.485,8.797$ & 0.326 \\
WBC & -0.055 & 0.234 & 0.946 & $0.595,1.498$ & 0.814 \\
CRP & -0.005 & 0.019 & 0.995 & $0.959,1.032$ & 0.789 \\
ESR & 0.038 & 0.045 & 1.039 & $0.951,1.135$ & 0.03 \\
PLT & 0.001 & 0.007 & 1.001 & $0.987,1.016$ & 0.849 \\
Gender & 0.508 & 2.323 & 29.377 & $0.310,2728.17$ & 0.146 \\
Age & 0.508 & 0.677 & 1.662 & $0.441,6.263$ & 0.453 \\
\hline
\end{tabular}

CAL, coronary artery lesion; $\mathrm{Cl}$, confidence interval; CRP, C-reaction protein; ESR, erythrocyte sedimentation rate; $\mathrm{KD}$, Kawasaki disease; $\mathrm{OR}$, odds ratio; $\mathrm{PLT}$, platelet count; WBC, white blood cell. capillary immune injury. More research is required into the exact pathophysiological mechanisms. The homeostasis of complement system activation and inactivation is regulated by the interaction between complement regulatory factor and complement component in vivo. CD59, a complement regulatory protein, regulates the formation and function of C5b-9 complex, preventing harm to cell lysis caused by the membrane attack complex. CD59 can also eliminate the immune complexes and promote phagocytosis of cells (25). Our study showed that plasma concentration of C5b-9 significantly lower in CAL. Maybe most C5b-9 was primarily deposits in the glomerulus and lead lower blood concentration (26). There may be an association with other increased complement regulatory proteins (CD35, CR1, CR2, and Crry) in vivo, which 
regulate immune status and inhibit complex formation, with C5b-9 in dynamic balance, but the specific biological mechanism needs further study.

Clinical and experimental data have shown that severe and persistent inflammation during the course of $\mathrm{KD}$ is a risk factor for CAL. CRP, a factor in systemic inflammatory response, is relevant to the prognosis of $\mathrm{KD}$ patients with CAL (27). Serum CRP level is increased in patients with myocardial ischemia and sudden death due to coronary artery disease (28). Previous studies have shown that elevated ESR in KD patients is disadvantageous to the prognosis of CAL (29). In our study, CRP was significantly higher in patients with CAL, and it was also revealed that PLT was significantly higher in KD patients with CAL, possibly related to vascular inflammation and activation of the coagulation system. Significant correlation between demographic factors, such as age and gender, and the recovery time of CAL, was not demonstrated.

Detection of the dynamic change of plasma MASP-1 concentration, along with other complement components during recovery from $\mathrm{KD}$, would provide more information, and this was a limitation of the study. Further research is required to verify the correlation between plasma MASP-1 concentration and the recovery time of $\mathrm{KD}$ patients with $\mathrm{CAL}$, for example, by establishment of a KD animal model and using coronary artery biopsy. In summary, our evidence concludes that lower plasma MASP-1 concentration during the early stage of $\mathrm{KD}$ is related to the earlier recovery time from CAL for patients older than $1 \mathrm{y}$, and this information provides an experimental basis for active intervention and future targeted therapies.

\section{METHODS \\ Subjects}

Eighty-five children, who had been hospitalized and newly diagnosed with KD between March 2011 and November 2013 at the Children's Hospital affiliated to the Capital Institute of Pediatrics, were enrolled. Their age ranged from 2 mo to $5 \mathrm{y}$ (mean age: $1.61 \pm 1.14 \mathrm{y}$ ). The ratio of males to females was 1.53:1. The standard diagnostic criteria for KD were used (30). None of the subjects had been previously diagnosed with $\mathrm{KD}$ and they had not received the standard treatment of intravenous immunoglobulin from the onset of fever. The research program complied with the Declaration of Helsinki and was approved by the Children's Hospital affiliated to Capital Institute of Pediatric Medical Ethics Committee. Informed consent was obtained from the parents of the children. On the basis of the results of two-dimensional echocardiography coronary examination, the subjects were divided into two groups: KD with CAL (referred to as the CAL group) $(n=37)$ and $\mathrm{KD}$ without CAL (referred to as the NCAL group) $(n=48)$. The CAL group was further divided into two subgroups according to the degree of CAL: mild CAL $(n=31)$ and moderate or severe CAL $(n=6)$. According to age, patients with CAL were divided into two subgroups, younger than 1 y group $(n=9)$ and older than 1 y group $(n=28)$. Patients with nonstandard therapy before hospitalization or a relevant history of cardiovascular disease were excluded.

All subjects were followed up once a month for 3 mo after discharge from the hospital. Follow-up included: echocardiography, electrocardiography, blood coagulation function, routine blood counts, and liver and kidney function tests. After the initial 3-mo follow-up, patients with coronary arteries that were normal or returning to normal had the follow-up extended to $3 \mathrm{mo}, 6 \mathrm{mo}$, or $1 \mathrm{y}$. Patients who continued to demonstrate coronary artery abnormality were followed up every 1 or 2 mo.

\section{Blood Sample Collecting and Processing}

Blood samples of all subjects were collected before the initiation of standard therapy within $2 \mathrm{wk}$ from the onset of fever. Morning fasting venous blood samples of $2 \mathrm{ml}$ were drawn and injected into a sealed tube, coated with $0.34 \mathrm{~mol} / \mathrm{l}$ ethylenediaminetetraacetic acid per $4.5 \mathrm{ml}$ blood sample. The samples were refrigerated immediately at $4{ }^{\circ} \mathrm{C}$ to prevent complement degradation ex vivo. After centrifugation of $3,000 \mathrm{rpm}$ for $15 \mathrm{~min}$, plasma was separated in a $200-\mu \mathrm{l}$ Eppendorf Tubes to avoid repeated freeze-thaw cycles during testing, and stored at $-80^{\circ} \mathrm{C}$.

\section{Detection of Plasma Complement, Inflammatory Markers, and}

\section{Coronary Artery Lesion}

Plasma MASP-1, C5b-9, and CD59 were measured using ELISA (Lot: L130903054; Uscn Life Science, Wuhan, China) with double holes set. Simultaneously, testing for CRP, ESR, WBC, and PLT was performed. Two-dimensional echocardiography (iE33 ultrasound system, Philips, The Netherlands) was used to evaluate the coronary artery, carried out by a cardiovascular specialist. During this procedure, children lay still in supine or the left lateral decubitus position. Children younger than 3 y who could not cooperate were administered with $10 \%$ chloral hydrate $0.5 \mathrm{mg} / \mathrm{kg}$ for sedation. The size of the inner diameters of the right main coronary artery, left main coronary artery, left anterior descending artery, and left circumflex artery were measured, and measurement was repeated three times for each position, with the mean taken as the ultimate value. For the CAL groups, dilatation or ectasia was descriptive term used to characterize an internal vessel diameter greater than $2 \mathrm{SD}$ of the average but less than $0.4 \mathrm{~cm}$; a diameter greater than $0.4 \mathrm{~cm}$ but less than $0.8 \mathrm{~cm}$ was defined as aneurysm; and giant aneurysms defined by an internal diameter greater than $0.8 \mathrm{~cm}$, and these subjects were assigned to one of three subgroups depending on the extent of the visualized CAL: mild (dilatation or ectasia), moderate (aneurysms), or severe (giant aneurysms).

\section{Statistical Analysis}

Data analysis was performed with SAS statistical software version 9.2 (SAS Institute, Cary, NC). Data were expressed as mean \pm SD for normal distributions and median (25th, 75th percentile) for non-normal distributions. Differences in quantitative parameters between groups were assessed using one-way ANOVA and least-significant difference $t$-test (for normally distributed data) or rank sum test (for non-normally distributed data). Spearman's correlation was used to determine the relationship between two non-normally distributed variables or between non-normally and normally distributed variables. Pearson's correlation was used to determine the relationship between two normally distributed variables. A multivariate logistic regression model was used to calculate odds ratio, 95\% confidence interval, and corresponding $P$ values. All $P$ values were two-sided, and $P<0.05$ was considered statistically significant.

\section{ACKNOWLEDGMENT}

We thank the families of the children for their active participation in this study.

\section{STATEMENT OF FINANCIAL SUPPORT}

No financial assistance was received to support this study.

Disclosure: There are no any financial ties to products in the study or potential/perceived conflicts of interest.

\section{REFERENCES}

1. Cummings C, McCarthy P, van Hoff J, Porter G Jr. Kawasaki disease associated with reactive hemophagocytic lymphohistiocytosis. Pediatr Infect Dis J 2008;27:1116-8.

2. Matsubara T, Ichiyama T, Furukawa S. Immunological profile of peripheral blood lymphocytes and monocytes/macrophages in Kawasaki disease. Clin Exp Immunol 2005;141:381-7.

3. Alexoudi I, Kanakis M, Kapsimali V, Vaiopoulos G. Kawasaki disease: current aspects on aetiopathogenesis and therapeutic management. Autoimmun Rev 2011;10:544-7. 
4. Kato H, Sugimura T, Akagi T, et al. Long-term consequences of Kawasaki disease. A 10- to 21-year follow-up study of 594 patients. Circulation 1996;94:1379-85.

5. Franco A, Shimizu C, Tremoulet AH, Burns JC. Memory T-cells and characterization of peripheral T-cell clones in acute Kawasaki disease. Autoimmunity 2010;43:317-24.

6. Meri S. Complement activation in diseases presenting with thrombotic microangiopathy. Eur J Intern Med 2013;24:496-502.

7. Megyeri M, Jani PK, Kajdácsi E, et al. Serum MASP-1 in complex with MBL activates endothelial cells. Mol Immunol 2014;59:39-45.

8. Ishikawa T, Iwashima S. Endothelial dysfunction in children within 5 years after onset of Kawasaki disease. J Pediatr 2013;163:1117-21.

9. Kaneko K, Savage CO, Pottinger BE, Shah V, Pearson JD, Dillon MJ. Antiendothelial cell antibodies can be cytotoxic to endothelial cells without cytokine pre-stimulation and correlate with ELISA antibody measurement in Kawasaki disease. Clin Exp Immunol 1994;98:264-9.

10. Cheung YF, Ho MH, Ip WK, Fok SF, Yung TC, Lau YL. Modulating effects of mannose binding lectin genotype on arterial stiffness in children after Kawasaki disease. Pediatr Res 2004;56:591-6.

11. Du ZD, Zhang T, Liang L, et al. Epidemiologic picture of Kawasaki disease in Beijing from 1995 through 1999. Pediatr Infect Dis J 2002; 21:103-7.

12. Makino N, Nakamura Y, Yashiro M, et al. Descriptive epidemiology of Kawasaki disease in Japan, 2011-2012: from the results of the $22^{\text {nd }}$ nationwide survey. J Epidemiol 2015;25:239-45.

13. Zhang C, Li Y, Wang C, et al. Complement 5a receptor mediates angiotensin II-induced cardiac inflammation and remodeling. Arterioscler Thromb Vasc Biol 2014;34:1240-8.

14. Nakamura A, Okigaki M, Miura N, et al. Involvement of mannose-binding lectin in the pathogenesis of Kawasaki disease-like murine vasculitis. Clin Immunol 2014;153:64-72.

15. Biezeveld MH, Kuipers IM, Geissler J, et al. Association of mannose-binding lectin genotype with cardiovascular abnormalities in Kawasaki disease. Lancet 2003;361:1268-70.

16. Biezeveld MH, Geissler J, Weverling GJ, et al. Polymorphisms in the mannose-binding lectin gene as determinants of age-defined risk of coronary artery lesions in Kawasaki disease. Arthritis Rheum 2006;54: $369-76$.

17. Megyeri M, Makó V, Beinrohr L, et al. Complement protease MASP-1 activates human endothelial cells: PAR4 activation is a link between complement and endothelial function. J Immunol 2009;183:3409-16.
18. Gulla KC, Gupta K, Krarup A, et al. Activation of mannan-binding lectinassociated serine proteases leads to generation of a fibrin clot. Immunology 2010;129:482-95.

19. Turner MW. Mannose-binding lectin: the pluripotent molecule of the innate immune system. Immunol Today 1996;17:532-40.

20. Frauenknecht V, Thiel S, Storm L, et al. Plasma levels of mannanbinding lectin (MBL)-associated serine proteases (MASPs) and MBLassociated protein in cardio- and cerebrovascular diseases. Clin Exp Immunol 2013;173:112-20.

21. Jani PK, Kajdácsi E, Megyeri M, et al. MASP-1 induces a unique cytokine pattern in endothelial cells: a novel link between complement system and neutrophil granulocytes. PLoS One 2014;9:e87104.

22. Printz BF, Sleeper LA, Newburger JW, et al.; Pediatric Heart Network Investigators. Noncoronary cardiac abnormalities are associated with coronary artery dilation and with laboratory inflammatory markers in acute Kawasaki disease. J Am Coll Cardiol 2011;57:86-92.

23. Orsini F, Villa P, Parrella S, et al. Targeting mannose-binding lectin confers long-lasting protection with a surprisingly wide therapeutic window in cerebral ischemia. Circulation 2012;126:1484-94.

24. Endo M, Ohi H, Ohsawa I, Fujita T, Matsushita M. Complement activation through the lectin pathway in patients with Henoch-Schönlein purpura nephritis. Am J Kidney Dis 2000;35:401-7.

25. Gorter A, Blok VT, Haasnoot WH, Ensink NG, Daha MR, Fleuren GJ. Expression of CD46, CD55, and CD59 on renal tumor cell lines and their role in preventing complement-mediated tumor cell lysis. Lab Invest 1996;74:1039-49.

26. Liu L, Qiu W, Wang H, et al. Sublytic C5b-9 complexes induce apoptosis of glomerular mesangial cells in rats with Thy- 1 nephritis through role of interferon regulatory factor-1-dependent caspase 8 activation. J Biol Chem 2012;287:16410-23.

27. Mitani Y, Sawada H, Hayakawa H, et al. Elevated levels of high-sensitivity C-reactive protein and serum amyloid-A late after Kawasaki disease: association between inflammation and late coronary sequelae in Kawasaki disease. Circulation 2005;111:38-43.

28. Auer J, Berent R, Lassnig E, Eber B. C-reactive protein and coronary artery disease. Jpn Heart J 2002;43:607-19.

29. Chen J, Liu Y, Liu W, Wu Z. A meta-analysis of the biomarkers associated with coronary artery lesions secondary to Kawasaki disease in Chinese children. J Huazhong Univ Sci Technolog Med Sci 2011;31:705-11.

30. Kuo HC, Yang KD, Chang WC, Ger LP, Hsieh KS. Kawasaki disease: an update on diagnosis and treatment. Pediatr Neonatol 2012;53:4-11. 